\title{
A Kinematically Beamed, Low Energy Pulsed Neutron Source for Active Interrogation
}

D. Dietrich, C. Hagmann, P. Kerr, L. Nakae, M. Rowland, N. Snyderman, W. Stoeffl, R. Hamm

October 11, 2004

Conference on Application of Accellerators in Research and Industry

Fort Worth, TX, United States

October 11, 2004 through October 15, 2004 
This document was prepared as an account of work sponsored by an agency of the United States Government. Neither the United States Government nor the University of California nor any of their employees, makes any warranty, express or implied, or assumes any legal liability or responsibility for the accuracy, completeness, or usefulness of any information, apparatus, product, or process disclosed, or represents that its use would not infringe privately owned rights. Reference herein to any specific commercial product, process, or service by trade name, trademark, manufacturer, or otherwise, does not necessarily constitute or imply its endorsement, recommendation, or favoring by the United States Government or the University of California. The views and opinions of authors expressed herein do not necessarily state or reflect those of the United States Government or the University of California, and shall not be used for advertising or product endorsement purposes. 


\section{EF-6 A Kinematically Beamed, Low Energy Pulsed}

\section{Abstract}

\section{Neutron Source for Active Interrogation}

Dan Dietrich, Chris Hagmann, Phil Kerr, Les Nakae, Mark Rowland, Neal Snyderman, and Wolfgang Stoeffl; Lawrence Livermore National Laboratory, and Robert Hamm, AccSys Technologies

We are developing a new active interrogation system based on a kinematically focused low energy neutron beam. The key idea is that one of the defining characteristics of SNM (Special Nuclear Materials) is the ability for low energy or thermal neutrons to induce fission. Thus by using low energy neutrons for the interrogation source we can accomplish three goals, 1) Energy discrimination allows us to measure the prompt fast fission neutrons produced while the interrogation beam is on; 2) Neutrons with an energy of approximately 60 to $100 \mathrm{keV}$ do not fission 238U and Thorium, but penetrate bulk material nearly as far as high energy neutrons do and 3) below about $100 \mathrm{keV}$ neutrons lose their energy by kinematical collisions rather than via the nuclear $(\mathrm{n}, 2 \mathrm{n})$ or $(\mathrm{n}, \mathrm{n}$ ') processes thus further simplifying the prompt neutron induced background. $60 \mathrm{keV}$ neutrons create a low radiation dose and readily thermal capture in normal materials, thus providing a clean spectroscopic signature of the intervening materials. The kinematically beamed source also eliminates the need for heavy backward and sideway neutron shielding. We have designed and built a very compact pulsed neutron source, based on an RFQ proton accelerator and a lithium target. We are developing fast neutron detectors that are nearly insensitive to the ever-present thermal neutron and neutron capture induced gamma ray background. The detection of only a few high energy fission neutrons in time correlation with the linac pulse will be a clear indication of the presence of SNM. 


\section{Introduction}

2 There are many possible scenarios for active interrogation of commercial cargo. Compared to

3 a classic nuclear physics laboratory environment, cargo scanning has to deal with an enormous

4 range in both the amount and makeup of attenuating or shielding materials. This gives rise to a

5 formidable problem of background discrimination making cargo inspection a very different

6 problem from measurements designed to measure nuclear properties in a clutter free controlled

7 environment. The main problem is the enormous thickness and variety of the possible

8 intervening material, approaching the thickness of nuclear reactor shielding walls. For some

9 scenarios, either gamma rays or neutrons cannot penetrate the cargo efficiently. On the other

10 hand, it is very difficult to shield both gamma (or X-rays) and neutron penetration at the same

11 time when there is a limit on the weight of the shielding. For gamma rays, heavy elements are

12 difficult to penetrate, for neutrons, light elements like plastic and water are difficult. The most

13 promising detection method that we can create is a combination of 3 different methods to cover

14 all possible scenarios:

16 1) Unshielded SNM like Uranium 235 (mixed with U238) or Pu239 and other SNM

17 materials are easily detected by passive radiation measurement with large gamma ray

18 scintillation detectors and neutron detectors.

19 2) Heavily shielded SNM in a low $Z$ neutron absorbing overburden is easily observed with a 20 high energy X-ray scan of the cargo, (preferably 2-axis and two different X-ray energies

21 for better material identification, like the luggage scanners used in airports). The

22 detection of a heavy and very dense object in the middle of a large amount of 


\section{Concept Description} shipping containers. interrogating neutrons.

\section{The neutron source} hydrogenous material will be very suspicious and is usually not encountered in normal

3) Active neutron interrogation of a container without a large amount of homogeneously distributed hydrogenous material can unmistakably detect the presence of SNM and serve to remove all confusion in interpretation of complex radiographs. The active interrogation needs to be exclusively sensitive and specific to SNM like 235U or 239Pu, and not confused by passive materials like Thorium, which is present in many materials at a significant level. The return signal of the active interrogation has to be unique to the presence of SNM, and should produce no signal from the many tons of "inert" material present in a typical container.

There is one unique method of interrogation which is very specific to SNM and produces an essentially background free return signal. Sending out low to medium energy neutrons in the energy range between 10 and $200 \mathrm{keV}$, and observing the induced 1 to $5 \mathrm{MeV}$ fission neutrons from SNM with detectors able to discriminate between photons, fission neutrons and

This method produces a nearly background free identification signal for SNM. Even a small number of detected fast neutrons will be a positive signal, since the fast neutron background from natural sources is very low.

Our source of medium energy neutrons is the $(p, n)$ reaction of a $2 \mathrm{MeV}$ proton beam on a 7-Li target[1,2]. Since the early days of nuclear physics it has been known that one can produce medium energy neutrons with the ${ }^{7} \operatorname{Li}(\mathrm{p}, \mathrm{n})$ reaction. But since there was little physics use for a 
1 medium energy neutron source, this reaction was rarely used and very few accelerators have

2 been built to make use of this reaction. The reaction has a threshold of $1.88 \mathrm{MeV}$ and the cross

3 section rises to its full value within $20 \mathrm{keV}$ of the threshold proton beam energy. It is a very

4 sharp threshold reaction. If one chooses a proton beam energy just above the reaction threshold,

5 it is possible to restrict the neutron emission pattern to a 60 -degree forward cone. The kinematics

6 of this reaction produces a forward directed neutron beam. There are no neutrons emitted

7 backwards from the target and the opening angle is controlled by the proton beam energy. The

8 higher the proton beam energy above threshold, the wider the opening emission angle will be.

9 The narrow opening angle reduces the neutron activation of the surrounding facilities

10 dramatically. There is also no need for bulky and heavy neutron shielding in order to "collimate"

11 the beam. Having no need for bulky shielding, one can place the fast neutron detectors rather

12 close to the accelerator and target. Since the outgoing neutrons have rather low energy, the

13 radiation dose delivered to the cargo is lower than an equivalent number of higher energy

14 neutrons and useable beams do not pose a threat to equipment or humans in the cargo.

15 Neutron production rates can be as high as $10^{10}$ per second into a 1 steradian cone,

16 equivalent to a ten times higher strength source emitting into $4 \pi$. With a strong source, one can

17 scan a complete cargo container in much less than 1 minute. The $2 \mathrm{MeV}$ proton accelerator is

18 less than half the size of a typical office desk, is portable, plugs into a regular electrical outlet

19 and requires no cooling water. There is the possibility to build a very tightly focused neutron

20 beam by reversing the ${ }^{7} \mathrm{Li}(\mathrm{p}, \mathrm{n})$ reaction to ${ }^{1} \mathrm{H}\left({ }^{7} \mathrm{Li}, \mathrm{n}\right)$. The benefit is a very narrow and high

21 brightness neutron beam, the drawback is that the accelerator to produce $14 \mathrm{MeV}^{7} \mathrm{Li}$ is much

22 larger and much more expensive. 


\section{$1 \quad$ Fast neutron detection}

The fast neutron sensitive detectors are a key to the nearly background free detection of

3 SNM. Sending out a high flux of neutrons into a random cargo will produce significant gamma

4 radiation, since most neutrons will eventually be captured resulting in the emission of very

5 energetic gamma rays. The typical neutron capture reaction releases about 7-8 MeV of gamma

6 ray energy. The detector has to be able to distinguish between the gamma rays and the energetic

7 neutrons. Discriminating liquid scintillator detectors were developed many years ago, and the

8 pulse shape discriminating read-out electronics has been steadily improved in the last 20 years.

9 The development was mainly driven by the development of low background detectors for deep

10 underground astro-physics instruments.

\section{Gamma ray - neutron discrimination}

12 Gamma ray - neutron discrimination is a strong function the actual count rate in the

13 detector, so it is beneficial to keep the absolute count rate rather low to eliminate loss of

14 discrimination due to pileup. Our current detector array is segmented to keep the individual

15 detectors to less than 1-liter volume per element and are about one square meter in total surface

16 area.. Arrays on each side of the neutron source and a few on the opposite sides of the container

17 will be sufficient. Our tests have shown that we have near zero background in our fast neutron

18 detectors, even while the interrogating neutron beam is on. This makes it possible to detect SNM

19 with only a few tens or hundreds of counted high-energy fission neutrons. We currently employ a

20 clever analog electronic circuit to distinguish the pulse shapes, but it performance degrades at

21 high-count rates. A fully digital readout will practically eliminate this problem and give a much

22 cleaner neutron signal, even in a high gamma rate environment. We plan to quantitatively assess

23 the improvement in overall system sensitivity by implementing a digital event readout system. 


\section{$1 \quad$ Neutron diffusion}

The free path length of fast neutrons in most materials is rather short, typically between 2

3 and $5 \mathrm{~cm}$. The free path length between elastic scatterings is surprisingly independent of atomic

4 mass, making most materials look the same for neutron penetration. Neutrons can scatter for

5 many meters in heavy material before they thermalize and are ultimately captured. An important

6 exception is hydrogenous material like polyethylene or water. Neutrons lose some energy in

7 every collision; the typical loss is proportional to the atomic weight ratio of the neutron and the

8 scattering nucleus. Thus, neutrons lose their energy comparatively fast in materials with a

9 significant fraction of hydrogen, like water or polyethylene. In water, the useful diffusion depth

10 is about $30 \mathrm{~cm}$. In heavy materials, a container full of tools or electronics is not an obstacle.

11 The $60 \mathrm{keV}$ neutrons will have a useful penetration depth comparable to a multi-MeV

12 neutron beam in non- hydrogenous materials. This is because most of the diffusion length comes

13 from the random walk of the ever-slowing neutrons at lower energies. The energy loss is an

14 exponential process, so very energetic neutrons rapidly slow down to medium energies, and then

15 undergo the same diffusion processes as the $60 \mathrm{keV}$ neutrons. At higher energies, there is also an

16 extra contribution to the energy loss mechanism since fast neutrons lose much of their energy by

17 inelastic excitation of the target nuclei, producing unwanted additional gamma radiation.

18 Thermalized neutrons will cause much of the fission of SNM in a cargo container, where

19 the fission cross-section is very large for $235 \mathrm{U}$ and $239 \mathrm{Pu}$. The fast fission neutrons with an

20 average energy of $2 \mathrm{MeV}$ have to be able to exit the container, reversing the path of the

21 interrogating neutrons. Only neutrons that do not lose too much energy on their way out can be

22 counted, since the area is flooded with low energy interrogating neutrons. 
Most neutrons will scatter in the cargo material until the neutron reaches thermal energy,

3 and then they undergo a capture reaction. Most bulk materials with very few exceptions have

4 very small capture cross-sections for energetic neutrons. The elastic scattering energy loss

5 mechanism depends strongly on the atomic mass of the material; in non-hydrogen bearing

6 material it takes hundreds or thousands of scattering reaction to reach thermal neutron energies.

7 The long random walk path of the neutron allows it to diffuse up to 1 meter without severe

8 attenuation. If large amounts of hydrogen are present, the neutrons can lose their energy much

9 faster and the penetration depth is reduced. But even fast neutrons lose part of their energy in the

10 first few collisions and then follow the same path as lower energy neutrons.

11 The penetration depths for different materials and energies were modeled with the Monte

12 Carlo code MCNP. We used semi-infinite slabs of polyethylene, borated polyethylene,

13 aluminum, and iron, with neutron energies of $0.06,2.0$, and $14.0 \mathrm{MeV}$ impinging at normal

14 incidence (see Table 1). The neutrons were transported until they either escaped or were lost

15 through capture. The simulations showed large (few meters) penetration depths for aluminum

16 and iron slabs almost independent of neutron energy. On the other hand, we noticed significant

17 differences in the penetration depths for hydrogenous materials. Due to the large proton cross

18 section at thermal and intermediate neutron energies, the effective penetration depth of 0.06

$19 \mathrm{MeV}$ neutrons is limited to $\sim 20 \mathrm{~cm}$.

Table 1, Penetration depths (defined as the depth reached by $1 \%$ of incident neutrons).

\begin{tabular}{|l|l|l|l|}
\hline & $\mathrm{E}=0.06 \mathrm{MeV}$ & $\mathrm{E}=2.0 \mathrm{MeV}$ & $\mathrm{E}=14.0 \mathrm{MeV}$ \\
\hline Polyethylene & 15 & 26 & 68 \\
\hline Borated Polyethylene & 8 & 22 & 64 \\
\hline $\mathrm{Al}$ & 269 & 257 & 266 \\
\hline $\mathrm{Fe}$ & 98 & 138 & 151 \\
\hline
\end{tabular}




\section{Neutron background}

2 The natural fast neutron background in the open environment is very low. Neutrons can

3 be generated by cosmic muon induced spallation reactions in the soil and atmosphere. The

4 typical muon flux at the surface of the earth is approximately $100 \mathrm{muon} / \mathrm{m}^{2} / \mathrm{sec}$, and the

5 associated fast neutron flux is about a factor 10 lower. If the interrogating neutron source is

6 pulsed, most of the natural background can be gated out, reducing the effective natural neutron

7 flux to less than 1 neutron $/ \mathrm{m}^{2} / \mathrm{sec}$. With a short measurement time, even a small number of

8 returned fast neutrons can indicate the presence of SNM. No other material can produce fast

9 neutrons when using medium energy neutrons as an interrogation tool. The threshold for $(\gamma, \mathrm{n})$

10 reaction on most materials is out of energy range for natural occurring radioactive elements. The

11 very few materials with low neutron producing reaction thresholds can easily be detected by

12 other means.

\section{Calibration}

15 Since the medium energy neutron interrogation technique is exclusively sensitive to

16 actual SNM nuclei, there is no substitute available for testing and calibrations. This raises an

17 interesting problem that one needs actual SNM material to test the operational performance of

18 the detection system. But low enriched SNM material (even a significant amount of D-38 at

$19 \quad 0.3 \%$ U235, will contain enough U-235 to be useful) is sufficient to test and calibrate the

20 detection system.

\section{Conclusions}

22 We have developed a working system for active neutron interrogation by selecting a

23 reaction that is very exclusive to the detection of SNM and is not compromised by natural 
1 background reactions. $60 \mathrm{keV}$ neutrons can penetrate all cargos of interest quite efficiently. The

2 exception is cargo with a high hydrogen content, but X-rays can easily penetrate such cargo, and

3 SNM would show up either through passive measurements or as a very substantial, high $\mathrm{Z}$ shield

4 in the midst of bulk hydrogenous material. Fast neutrons are only produced by SNM material,

5 normal cargo does not produce any background. Detecting fast neutrons on both sides of the

6 cargo gives a clean signal, where the detection of even a few dozen beam times correlated fast

7 neutrons is enough for a clean detection. We have demonstrated that the fast neutron detection

8 system is insensitive to the interrogation medium energy neutron beam. This allows us to

9 measure the fast neutron return signal while the interrogation beam is on, using the full intensity

10 of the fast fission neutrons produced. The system is insensitive to ${ }^{238} \mathrm{U}$ and Thorium that is

11 always present in significant amounts in all materials. It is also insensitive to all other non-SNM

12 material. Used in conjunction with passive measurements and low dose radiography, this

13 technique can assure a very high detection probability with an acceptable false alarm rate and

14 can form one leg of an inspection regime flexible enough to be applied to essentially all

15 incoming commerce regardless of form factor.

\section{Acknowledgements}

This work was performed under the auspices of the U.S. Department of Energy by

18 the University of California, Lawrence Livermore National Laboratory under Contract W-7405-

19 Eng-48, as work for others funded by the U.S. Department of Homeland Security. 


\section{$1 \quad$ References}

2 1. Charles L. Lee, "The Design of an Intense Accelerator-Based Epithermal Neutron Beam Prototype for BNCT Using Near-Threshold Reactions", PhD Thesis, Department of Nuclear Engineering, Massachusetts Institute of Technology, 1998.

5 2. Chris A. Hagmann, "Detecting Fissile Material by Neutron Irradiation with a

${ }^{7} \operatorname{Li}(\mathrm{p}, \mathrm{n}){ }^{7}$ Be Source”, UCRL-ID-153351, 2003. 